\title{
LA INVESTIGACIÓN EN EL DEPARTAMENTO DE PERIODISMO I
}

\author{
Ángel Acosta Romero \\ Carmen Espejo Cala \\ (Universidad de Sevilla) \\ http://dx.doi.org/10.12795/AdMIRA.2009.01.15
}

El Departamento de Periodismo I de la Universidad de Sevilla se constituye oficialmente en el verano del año 2003 tras el proceso de fragmentación en dos departamentos del primitivo y único Departamento de Periodismo existente hasta ese momento en la Facultad de Comunicación (inicialmente de Ciencias de la Información) de la Universidad Hispalense. En esa nueva unidad departamental se integran voluntariamente un grupo de profesores universitarios que, dentro de un amplio espectro de preocupaciones teóricas, metodológicas y prácticas referidas al Área de investigación "Periodismo", comparten una visión crítica de la sociedad y de los medios de comunicación, y pretenden llevar a cabo, en su ámbito propio, proyectos de investigación encaminados a la ampliación del conocimiento, a la promoción del saber y a la socialización de los beneficios y resultados de la actividad investigadora tanto en su entorno geográfico más cercano como a nivel nacional e internacional.

Así, por ejemplo, desde sus inicios, el Departamento de Periodismo I ha mostrado una clara orientación hacia la cooperación y el intercambio con universidades y centros de investigación latinoamericanos, una vocación coherente con la historia, legado y proyección internacional de la propia Universidad de Sevilla, y que se ha visto plasmada en estos años por un buen número de proyectos y publicaciones de carácter científico que además contienen objetivos relacionados con el estrechamiento de vínculos de cooperación solidaria y extensión de principios y valores éticos y políticos en defensa activa de la justicia social, la defensa de los derechos humanos, de la paz y del desarrollo ecológicamente sostenible. Todo ello en el marco de las políticas culturales y de los procesos de comunicación que tejen y transforman los valores y universos simbólicos de nuestro presente globalizado.

Este espíritu fundacional del departamento es transmitido por el profesorado a los estudiantes en todos los niveles de la formación universitaria y desde luego tiene su manifestación concreta en las labores investigadoras que a nivel individual o a través de diferentes instancias colectivas (grupos de investigación, seminarios, revista, futuro master...) desarrollan los actuales integrantes del departamento.

Para ordenar de alguna forma el material que hemos podido recabar de los profesores para la redacción de esta reseña, que nos fue solicitada por la profesora Virginia Guarinos para un volumen conmemorativo de los primeros veinte años de vida de la Facultad de Comunicación que se celebran en 2009, podríamos haber optado por un sistema de clasificación basado en las constantes investigadoras de los grupos de investigación oficiales cuyos responsables pertenecen al Departamento, o en las Academias en las que se organiza la dedicación docente, o incluso en las líneas de investigación marcadas en el antiguo Programa de Doctorado en cuyo marco, por cierto, se han leído once tesis doctorales en estos años, aunque se encuentra ya extinto a la 
espera de la puesta en marcha de los nuevos postgrados adaptados al Espacio Europeo de Educación Superior; pero, por razones de claridad expositiva, hemos preferido agrupar las principales aportaciones investigadoras de los profesores del Departamento en distintas líneas temáticas que se irán señalando en los párrafos siguientes, eso sí aludiendo a los distintos Grupos de Investigación y otras instancias de investigación en las que participan directa o indirectamente, y todo ello atendiendo a la propia información suministrada por los profesores.

En concreto, podemos distinguir, pero no separar, distintas líneas específicas o trayectorias investigadoras fundamentales que son las referidas a "Documentación y exomemoria", "Políticas comunicativas y cambio social", "Historia general de la comunicación y la propaganda", "Análisis del discurso y periodismos especializados", "Tecnología de la información y la comunicación" y "Teoría y epistemología de la comunicación y la información". Cabe insistir en que a pesar de que estas etiquetas temáticas nos ayudan a clasificar e identificar, en lo fundamental, las distintas líneas investigadoras del Departamento, la mayoría de ellas tienen un claro talante transversal que atraviesa las citadas fronteras temáticas y les otorga una dimensión enriquecedora en el ámbito general de los estudios sobre comunicación, cultura y mediación que definen a su vez la filosofía y la política científica del departamento.

Iniciamos este recorrido, aludiendo a la línea de investigación que tiene como marco de referencia básico el área de Documentación. De esta forma, la preocupación por desentrañar las relaciones entre "memoria", "cultura", "identidad" y "digitalidad", ha sido el objetivo del profesor Antonio García Gutiérrez, Catedrático de Documentación y actual director del Departamento, en algunas de sus últimas publicaciones, como por ejemplo: La memoria subrogada. Mediación, cultura y conciencia en la red digital (Granada, Universidad, 2002), Otra memoria es posible. Estrategias descolonizadoras del archivo mundial (Buenos Aires, La Crujía, 2004), Fijaciones. Estudios críticos sobre politicas, culturas y tecnologías de la memoria (Madrid, Biblioteca Nueva, 2005), Desclasificados: pluralismo lógico y violencia de la clasificación (Barcelona, Anthropos, 2007) y La identidad excesiva (Madrid, Biblioteca Nueva, 2009). En todas estas obras el profesor García Gutiérrez se sitúa bajo la perspectiva de una crítica radical al poder, a la lógica dominante y al capitalismo, así como en una apuesta hacia la emancipación individual y social.

En el mismo territorio de la memoria colectiva y la documentación en comunicación se encuentran los trabajos de Ángeles López Hernández, Profesora Titular y miembro del Grupo de Investigación en "Comunicación y Cultura", como lo demuestran sus múltiples artículos en revistas especializadas y sus monografías La selección de documentos. Problemas y soluciones desde una perspectiva metodológica y el manual Introducción a la Documentación Audiovisual. El grupo de profesores especialmente orientados al ámbito de la documentación lo completa la profesora Irene Tenorio, que cuenta en su haber con varias publicaciones en ese campo.

Otra de las líneas de investigación del departamento tiene que ver con la "Comunicación, Política y Cambio Social" (COMPOLÍTICAS), temática que da título al Grupo de investigación que dirige el profesor Francisco Sierra Caballero, actual Decano de la Facultad de Comunicación; en esta línea se plantean análisis y estudios especializados en el terreno teórico e histórico-crítico de los procesos de desarrollo y 
cambio social en torno a las mediaciones simbólicas e infocomunicacionales. Una muestra de este planteamiento es el volumen Culturas de guerra. Medios de información y violencia simbólica (Madrid,Cátedra, 2004) que el profesor Sierra ha publicado junto a Fernando Contreras Medina, también profesor del Departamento.

El terreno de la "Historia General de la Comunicación" es uno de los principales ejes vertebradores de la investigación en el Departamento de Periodismo I de la Universidad de Sevilla y en él destacan los múltiples trabajos desarrollados por la profesora Carmen Espejo Cala, que ha dedicado una especial atención a la Historia del Periodismo y, particularmente, a la historia del periodismo sevillano y andaluz de la Edad moderna y contemporánea; algunos de sus trabajos en revista más destacados son los artículos "Spanish news pamphlets on the 1755 earthquake: trade strategies of the printers of Seville" (SVEC, Oxford, 2005), y "El impresor sevillano Juan Gómez de Blas y los orígenes de la prensa periódica. La Gazeta Nueva de Sevilla (1661-1667)(ZER, Bilbao, 2008). Entre sus publicaciones en libro puede destacarse Propaganda impresa y construcción del Estado moderno y contemporáneo (Sevilla, Alfar, 2000), volumen colectivo coordinado por la profesora Espejo en el que también participa el profesor Ángel Acosta. Ha colaborado en volúmenes como Senderos de ilusión. Lecturas populares en Europa y América latina (Del siglo XVI a nuestros días), en Antonio Castillo Gómez, dir., (Gijón, Trea, 2007); La comunicación durante la Segunda República y la Guerra Civil (Zaragoza, Fragua, 2007); ABC de Sevilla, un Diario y una Ciudad (Sevilla, Universidad, 2007) y Relaciones de sucesos en la BUS. Antes de que existiera la prensa (Sevilla, Biblioteca Universitaria, 2009); de estos tres últimos volúmenes es además editora científica. En el ámbito del estudio de la prensa antigua se encuadra asimismo su participación como investigadora en el Proyecto I+D "Biblioteca Digital Siglo de Oro" y los convenios correspondientes suscritos con bibliotecas sevillanas.

Como se ha indicado, el profesor Ángel Acosta Romero, Titular de Teoría de la Comunicación, también ha dedicado una parte de su trabajo investigador en estos años a la labor de recuperación y edición de la obra de figuras destacadas de la historia del periodismo andaluz y sevillano; concretamente ha editado y prologado en tres volúmenes las Obras Completas de José $M^{a}$ Requena, novelista, poeta y reconocido periodista sevillano, personaje al que ha dedicado asimismo una monografía titulada Vida y obra de José María Requena (Sevilla, Alfar, 2001).

La propaganda, como fenómeno moderno de comunicación, ha sido el objetivo, tanto teórico como histórico, de diferentes trabajos y proyectos de investigación de los profesores del Departamento, entre ellos, del profesor Miguel Vázquez Liñán, miembro del grupo COMPOLÍTICAS y coordinador del Observatorio Eurasia dedicado al estudio, investigación y difusión de los principales fenómenos políticos y comunicacionales que tienen lugar especialmente en el espacio geográfico de la antigua Unión Soviética. Fruto de estas investigaciones son, por ejemplo, la monografía Desinformación y propaganda en la guerra de Chechenia (Sevilla, Padilla, 2005), así como varios artículos en revistas especializadas, y otros trabajos centrados en la propaganda relacionada con otros capítulos de la historia de España o en otros contextos internacionales como el movimiento zapatista en México, tema éste también tratado por el profesor Francisco Sierra en Comunicación e Insurgencia. La información y la propaganda en la guerra de Chiapas (Guipúzcoa, Iru, 1997). La labor investigadora del 
profesor Vázquez Liñán se completa con una serie de trabajos dedicados a una revisión teórica y metodológica sobre la propaganda, por ejemplo en: La construcción del consenso. Revisitando el modelo de propaganda de Noam Chomsky y Edward S. Herman (2006).

Recogiendo, en parte, el testigo del profesor Manuel Bernal Rodríguez, ya jubilado, otros jóvenes profesores del departamento se han especializado en la docencia y la investigación centrada en la redacción periodística y en el análisis del discurso y de los géneros periodísticos; tal es el caso del profesor Juan Carlos Gil que ha prestado especial atención a la crónica de temática taurina, objeto de varios trabajos publicados en revistas especializadas y de su Tesis Doctoral, publicada con el título Evolución histórica y cultural de la crónica taurina. De las primitivas reseñas a la crónica impresionista (Madrid, Siranda, 2007). El profesor Gil es actualmente Director de la Cátedra Ignacio Sánchez Mejías de Comunicación y Tauromaquia de reciente creación en la Universidad de Sevilla.

Algunos profesores del Departamento orientan su docencia e investigación a diversos ámbitos del periodismo especializado y de los gabinetes de comunicación y de las relaciones públicas. Es el caso del profesor Alejandro Antona Illanes, autor de una monografía y otros trabajos sobre Gabinetes de comunicación y el profesor Mariano Belenguer Jané, especialista en el periodismo de viajes.

Por su parte, el profesor Rafael González Galiana es Director del Grupo de Investigación en Comunicación y Cultura, al que pertenece también el joven investigador Francisco Baena Sánchez. Se trata de un equipo pluridisciplinario de estudio y formación en comunicación constituido como red de intercambio académico orientada al análisis de los cambios y estructuras significativas del sector de la información y la comunicación pública, desde un enfoque abierto e integrador que trata de vincular la teoría y la práctica, el pensamiento y la dinámica cultural, desde una visión crítica y una cultura de investigación productiva a la hora de plantear las problemáticas y propuestas de análisis sobre los objetos y lógicas que atraviesan los procesos comunicacionales.

Las nuevas tecnologías de la información y la comunicación y sus implicaciones y aplicaciones en todos los ámbitos sociales son también una prioridad en las líneas de investigación del Departamento, como lo muestra la existencia de un grupo de investigación dirigido por el profesor Fernando Contreras Medina; concretamente se trata del Grupo de Investigación en Tecnología de la Representación, Arte y Antropología Visual, que trabaja en Estudios Culturales, Medios e Innovación Tecnológica. y se ha especializado en el desarrollo de proyectos que abarcan desde la tecnología de la información, la cultura mediterránea e iberoamericana, hasta la ecología, el medio ambiente y el avance de los medios de divulgación de la ciencia. A este Grupo de Investigación pertenecen los profesores José Luis Campos García y Enrique Díaz León. El primero ha publicado diversos libros y artículos relacionados con el estudio cultural de las tecnologías de información y comunicación, entre ellos: Cuando la música cruzó la frontera digital. Aproximación al cambio tecnológico y cultural de la comunicación digital (Madrid, Biblioteca Nueva, 2008), Tecnologías de la información: una guía rápida para las empresas multimedia (en colaboración, Sevilla, Universidad, 2007). Por su parte, Enrique Díaz León desarrolla su labor 
investigadora en sintonía con su propia trayectoria profesional como responsable, desde hace ocho años, del programa de divulgación científica "El observatorio" de Canal Sur Radio, lo que le ha permitido publicar, entre otros, los trabajos: Andalucia, una historia de ciencia (Ciencia Digital, 2004), Un paseo por la Sevilla Cientifica e Industrial (2005) y Cádiz, entre la ciencia y la leyenda (2007). Algo semejante en relación con la conciliación entre tareas profesionales e investigadoras cabe decir del profesor asociado Pablo Juliá Juliá, reputado fotoperiodista y actualmente Director del Centro Andaluz de Fotografía, o del profesor Agustín Olmo, profesional de la televisión, así como de Miguel Alcíbar, prolífico actor y especialista en las relaciones entre ciencia y sociedad.

Una última línea de investigación que identifica claramente al Departamento de Periodismo I es la reflexión teórica y epistemológica en el ámbito de la comunicación y de la información. Uno de los profesores que representa este grupo es Ángel Acosta Romero, miembro del Grupo de Investigación interdepartamental en Teoría y Tecnología de la Comunicación y Director de la revista Discurso, órgano de difusión científica de la Asociación Andaluza de Semiótica. El profesor Acosta es especialista en Teoría de la Comunicación y en pensamiento complejo, temas sobre los que ha publicado más de una veintena de artículos en diversas revistas especializadas y sobre los que prepara un volumen monográfico actualmente en prensa. A la misma línea de investigación cabe asignar los numerosos trabajos publicados por la profesora $\mathrm{M}^{\mathrm{a}}$ del Mar Llera Llorente y por el profesor Ismael Roldán Castro.

En este grupo de teoría y epistemología de la comunicación, se incluye asimismo el amplio currículo investigador del profesor Francisco Sierra Caballero, actual Decano de la Facultad de Comunicación. Dirige el Centro Iberoamericano de Comunicación y la Revista de Estudios para el Desarrollo Social de la Comunicación, REDES.COM. Experto en políticas de comunicación, nuevas tecnologías y participación ciudadana de la Unión Europea, es autor de numerosos estudios, monografías y ensayos en materia de comunicación y desarrollo, políticas de comunicación y teoría de los medios, entre otros: Elementos de Teoría de la Información (Sevilla, MAD, 1999), Introducción a la Teoría de la Comunicación Educativa (Sevilla, MAD, 2000), Comunicación, educación y desarrollo. Apuntes para una historia de la comunicación educativa (Sevilla, Comunicación Social Ediciones, 2001), Los profesionales del silencio: la información y la guerra en la doctrina de EE.UU (Guipúzcoa, Iru, 2002), Bases de la política audiovisual europea (Sevilla, Mergablum, 2002), Politicas de comunicación y educación. Crítica y desarrollo de la sociedad del conocimiento (Barcelona, Gedisa, 2006). El profesor Sierra es en la actualidad fundador y miembro de la Junta Directiva de la Asociación Española de Investigación en Comunicación y miembro del Consejo Asesor de la RTVE, dependiente del Parlamento de Andalucía.

Una vez revisadas las principales líneas de investigación y aportaciones científicas de los integrantes del Departamento de Periodismo I, con la lógica brevedad que exije un informe de este tipo, queremos cerrar este reseña con una breve referencia a la revista del departamento, I/C (Información y Comunicación) que nació con el claro propósito de servir de canal de divulgación para investigadores y pensadores de la comunicación social. En las páginas de sus hasta este momento cinco número publicados, esta publicación trata de compaginar una declarada vocación científica con las intenciones de servir de plataforma de proyección de nuevas perspectivas, nuevos acercamientos y posturas de académicos y pensadores de reconocido prestigio y jóvenes investigadores 
contribuyendo al análisis, la reflexión, el debate y la crítica teórica de aquellos de aquellos para los que la comunicación no se restringe a un conjunto de técnicas y saberes profesionales. Editada por el Secretariado de Publicaciones de la Universidad de Sevilla, $I / C$ es un anuario abierto a las aportaciones de múltiples disciplinas y campos de conocimiento que se encuentran con el hecho socio-comunicativo en su matriz epistemológica. En sus páginas tienen cabida el estudio y la discusión de muy distintos intelectuales (filósofos, sociólogos, filólogos, historiadores, economistas...) implicados en los inclasificables matices de la información y la comunicación pública moderna, desde una decidida vocación innovadora. El contenido y política editorial del anuario presta, no obstante, preferente atención a las áreas, materias y subcampos de la investigación comunicológica tanto básica como aplicada.

Como se ha podido comprobar, el Departamento de Periodismo I de la Facultad de Comunicación de la Universidad de Sevilla ha desarrollado y sigue desarrollando una amplia y diversa actividad investigadora y de divulgación científica en el campo de las ciencias de la información y de la comunicación; esa diversidad, manifestada en las distintas líneas temáticas, conserva sin embargo una profunda coherencia e identidad derivadas de una filosofía y planteamiento común y radical que entiende la cultura como matriz de un campo problemático de investigación y crítica social que, en estos momentos más que nunca, tiene al conocimiento y a la comunicación como eje de acercamiento a los problemas de las sociedades contemporáneas. 